\title{
OPEN Mechanical power normalized to lung-thorax compliance indicates weaning readiness in prolonged ventilated patients
}

Alessandro Ghiani $\circledast^{1}{ }^{\bowtie}$, Joanna Paderewska ${ }^{1}$, Swenja Walcher ${ }^{1}$, Konstantinos Tsitouras $^{1}$, Claus Neurohr ${ }^{1,3}$ \& Nikolaus Kneidinger ${ }^{2,3}$

Since critical respiratory muscle workload is a significant determinant of weaning failure, applied mechanical power (MP) during artificial ventilation may serve for readiness testing before proceeding on a spontaneous breathing trial (SBT). Secondary analysis of a prospective, observational study in 130 prolonged ventilated, tracheotomized patients. Calculated MP's predictive SBT outcome performance was determined using the area under receiver operating characteristic curve (AUROC), measures derived from $k$-fold cross-validation (likelihood ratios, Matthew's correlation coefficient [MCC]), and a multivariable binary logistic regression model. Thirty (23.1\%) patients failed the SBT, with absolute MP presenting poor discriminatory ability (MCC 0.26; AUROC $0.68,95 \% \mathrm{Cl}[0.59-0.75]$, $p=0.002)$, considerably improved when normalized to lung-thorax compliance ( $\mathrm{LTC}_{\mathrm{dyn}}-\mathrm{MP}, \mathrm{MCC}$ 0.37 ; AUROC $0.76,95 \% \mathrm{CI}[0.68-0.83], p<0.001$ ) and mechanical ventilation $\mathrm{P}_{\mathrm{a}} \mathrm{CO}_{2}$ (so-called power index of the respiratory system $\left[\mathrm{PI}_{\mathrm{rs}}\right]$ : MCC $0.42 ;$ AUROC $\left.0.81[0.73-0.87], p<0.001\right)$. In the logistic regression analysis, $\mathrm{PI}_{\mathrm{rs}}\left(\mathrm{OR} 1.48\right.$ per $1000 \mathrm{~cm} \mathrm{H}_{2} \mathrm{O}^{2} / \mathrm{min}, 95 \% \mathrm{Cl}[1.24-1.76], p<0.001$ ) and its components $\mathrm{LTC}_{\mathrm{dyn}} \mathrm{MP}\left(1.25\right.$ per $\left.1000 \mathrm{cmH}_{2} \mathrm{O}^{2} / \mathrm{min},[1.06-1.46], p<0.001\right)$ and mechanical ventilation $\mathrm{P}_{\mathrm{a}} \mathrm{CO}_{2}(1.17[1.06-1.28], p<0.001)$ were independently related to SBT failure. MP normalized to respiratory system compliance may help identify prolonged mechanically ventilated patients ready for spontaneous breathing.

Mechanical ventilation, the core characteristic of intensive care, is a life-saving procedure for patients presenting severe respiratory failure. However, since prolonged ventilation is associated with increased morbidity and mortality ${ }^{1,2}$, liberation from the ventilator should begin as early as possible. A two-step strategy towards extubation, involving readiness testing and subsequent spontaneous breathing trials (SBT), has been shown to reduce the length of ventilation and costs of medical care in the $\mathrm{ICU}^{3}$.

So far, apart from clinical factors (e. g., evidence of clinical improvement, P/F ratio, adequate hemodynamics), several prediction variables (e.g., maximum inspiratory pressure, tidal volume, or minute ventilation) have been assessed in a one-time measurement for readiness testing ${ }^{4-6}$, with most of these traditional weaning predictors used to distinguish between patients who can or cannot sustain a trial of spontaneous breathing usually determined after a short period of self-ventilation following disconnection from the ventilator ${ }^{4}$. Serial measurement of esophageal pressure at the beginning of the SBT, reflecting patients' respiratory effort and breathing work, has improved further diagnostic accuracy in predicting weaning failure ${ }^{7}$, most probably because it reflects the underlying pathophysiology, namely an imbalance between respiratory impedance and neuromuscular capacity ${ }^{8,9}$. However, esophageal pressure measurement for assessment of patients' work of breathing, due to its complexity, is rarely used in daily routine. Moreover, not total power output (pressure times volume per time unit) but critical stress imposed on respiratory muscles (e. g. expressed as pressure-time product or tension-time index of the diaphragm) seems to be the major determinant of weaning failure ${ }^{8,10}$.

${ }^{1}$ Lung Center Stuttgart - Schillerhoehe Lung Clinic (Affiliated to the Robert-Bosch-Hospital GmbH, Stuttgart), Department of Pulmonology and Respiratory Medicine, Solitudestrasse 18, 70839 Gerlingen, Germany. ${ }^{2}$ Department of Internal Medicine V, Ludwig-Maximilians-University (LMU) of Munich, Munich, Germany. ${ }^{3}$ Comprehensive Pneumology Center (CPC-M), Member of the German Center for Lung Research (DZL), Munich, Germany. ${ }^{\square}$ email: alessandro.ghiani@rbk.de 


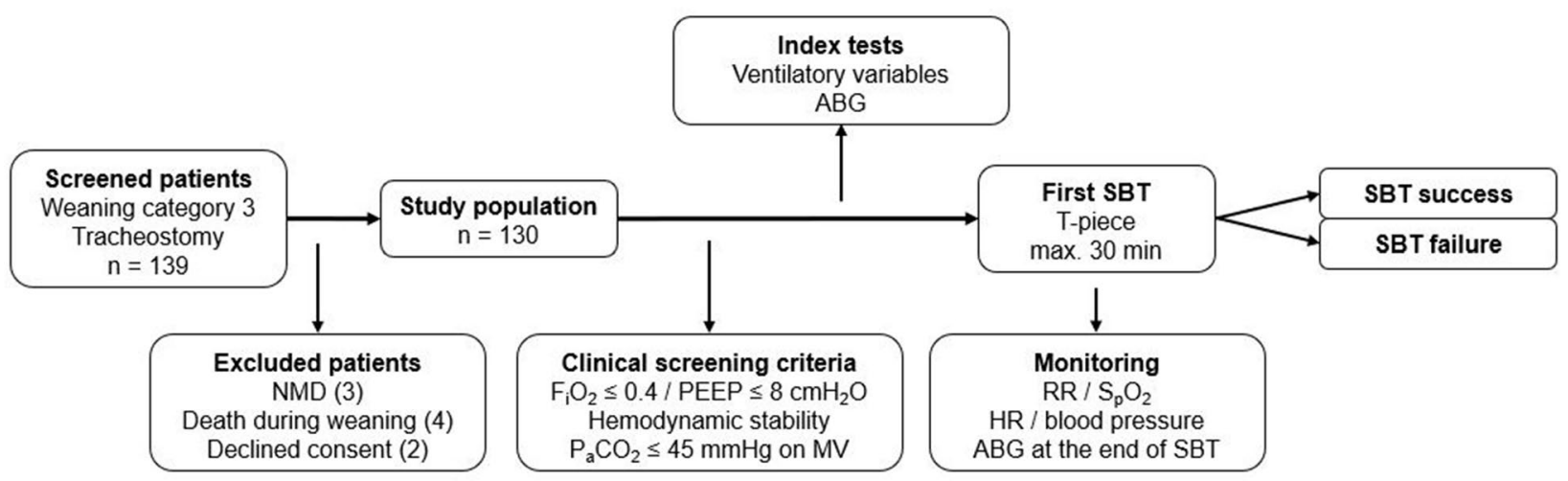

Figure 1. Study flow. Abbreviations: NMD, neuromuscular disease; $\mathrm{F}_{\mathrm{i}} \mathrm{O}_{2}$, fractional inspired oxygen; PEEP, positive end-expiratory pressure; $\mathrm{MV}$, mechanical ventilation; $\mathrm{ABG}$, arterial blood gas analysis; MP, mechanical power; SBT, spontaneous breathing trial; $\mathrm{RR}$, respiratory rate; $\mathrm{HR}$, heart rate.

Mechanical power (MP) of artificial ventilation, the energy transferred to the respiratory system per time unit, is a chief determinant of adequate gas exchange. Basically, the MP concept was introduced to shed further light on the underlying mechanisms behind ventilator-induced lung injury, as it unifies all ventilatory variables deemed responsible ${ }^{11}$. However, MP may also indicate respiratory muscle workload during spontaneous breathing, contributing significantly to weaning failure ${ }^{7-9}$. Accordingly, in a retrospective analysis, mechanical power normalized to lung-thorax compliance, a surrogate of applied power per unit of ventilated lung volume (consistent with stress intensity), was independently associated with the outcome of a spontaneous breathing trial $^{12}$.

In this analysis, we evaluated the discriminatory performance of mechanical power in predicting the outcome of a short weaning trial and thus indicate weaning readiness.

\section{Methods}

Secondary analysis of a previously reported prospective observational cohort study conducted at a specialized weaning center that focused on predictors of prolonged ventilation weaning failure ${ }^{13}$. The study was performed following the Declaration of Helsinki and approved by the local institutional review board for human studies (Ethics Committee of the State Chamber of Physicians of Baden-Wuerttemberg, Germany, file number F-2018116). All patients or a legal representative gave their written informed consent to participate. We now report MP as a candidate predictor of the first spontaneous breathing trial upon patient admission to our center.

Patient selection. Only tracheotomized patients referred for prolonged weaning, classified as Category 3 defined by Boles and co-workers ${ }^{14}$, were analyzed. Patients were excluded from the study if they had a diagnosis of neuromuscular disease, died before weaning completion, or declined consent (Fig. 1).

Spontaneous breathing trials. All patients were ventilated in the pressure-controlled, assist-control (A/C) mode (Vivo 50 \& 60, Breas Medical AB, Moelnlycke, Sweden). The main characteristic of this ventilation mode is that the same (high) pressure is applied during both an assisted and controlled breath, consistent with full ventilator support intending to defend alveolar ventilation and minimizing respiratory muscle activity, thereby recovering from the imposed work of breathing between SBT.

The first SBT upon admission to the weaning center occurred as soon as pre-defined clinical screening criteria for weaning readiness were met, which were (1) adequate oxygenation $\left(\mathrm{S}_{\mathrm{p}} \mathrm{O}_{2} \geq 92 \%\right)$ with fractional inspired oxygen $\leq 0.40$ and $\mathrm{PEEP} \leq 8 \mathrm{cmH}_{2} \mathrm{O}$, (2) hemodynamic stability without the need for vasopressors or inotropic agents, and (3) normocapnia $\left(\mathrm{P}_{\mathrm{a}} \mathrm{CO}_{2} \leq 45.0 \mathrm{mmHg}\right)$ on mechanical ventilation. Patients were not sedated when they underwent the SBT.

In general, the first SBT occurred in the early morning. Patients were placed in the semi-recumbent position for these trials. A few minutes before SBT started, ventilatory variables were obtained from the ventilators' display in a one-time measurement, and an arterial blood gas analysis (ABG) was performed simultaneously. The patient was then disconnected from the ventilator and breathed room air for $30 \mathrm{~min}$ through a T-piece with oxygen admixture at the same level as during mechanical ventilation. The first SBT was always performed under the supervision of a respiratory therapist, and vital signs were continuously monitored to detect respiratory distress immediately. Another ABG was performed at the end of SBT.

Candidate predictors of weaning. The following parameters were calculated from ventilatory variables and the corresponding arterial blood gas values collected immediately before the first SBT (Fig. 1): $\Delta \mathrm{P}_{\mathrm{aw}}$ (dynamic driving pressure, defined as $\mathrm{P}_{\max }$ - PEEP in the pressure-controlled ventilation mode), dynamic lung-thorax compliance $\left(\text { LTC }_{\text {dyn }}\right)^{15}$, mechanical power ${ }^{11}$ using the simplified formula proposed by Becher and colleagues ${ }^{16,17}$, and ventilatory ratio (VR, a surrogate for dead space ventilation $)^{18}$.

Total MP was further normalized to (1) the predicted body weight (a surrogate of the total lung capacity of a healthy individual, PBW-MP) ${ }^{19}$ and (2) LTC $_{\text {dyn }}$ (indicating actual ventilated lung volume, LTC $\left._{d y n}-M P\right)^{12}$. To allow comparability between individual respiratory rate and ventilator pressure settings, $\mathrm{LTC}_{\mathrm{dyn}}$-MP was ultimately 
corrected for corresponding mechanical ventilation $\mathrm{P}_{\mathrm{a}} \mathrm{CO}_{2}$ (simulating isocapnic conditions), called the Power index of the respiratory system ${ }^{13}$. Further details can be found in the Supplementary information.

Classification of outcome. Failure of the SBT was defined as the occurrence of objective clinical signs of respiratory failure (breathing frequency $>35 / \mathrm{min}$, tachycardia $>130 \mathrm{bpm}$, systolic blood pressure $>160 \mathrm{mmHg}$, or $\mathrm{S}_{\mathrm{p}} \mathrm{O}_{2}<88 \%$ despite increasing oxygen admixture) and/or changes in blood gas values consistent with ventilatory failure (hypercapnia $\left[\mathrm{P}_{\mathrm{a}} \mathrm{CO}_{2}>45.0 \mathrm{mmHg}\right]$ with or without respiratory acidosis $\left.[\mathrm{pH}<7.35]\right)^{14}$.

Statistical analysis. Descriptive and frequency statistics were used to summarize patients' demographics and baseline characteristics. As appropriate, differences between groups in categorical variables were analyzed using Chi-square or Fisher's exact tests. Continuous variables were subjected to the Kolmogorov-Smirnov normality test for homogeneity of variance. Depending on the statistical distribution, either Student's $t$-test or Mann-Whitney $U$-test was used to examine differences in these parameters.

Discriminatory weaning outcome ability of the selected variables was assessed through a receiver operating characteristic (ROC) curve analysis with diagnostic accuracy expressed as area under the ROC curve (AUROC). Moreover, we conducted a prospective, stratified, 2-times repeated, 2-fold cross-validation ${ }^{20}$ (Supplementary Fig. S1, Fig. S2, and Table S1), with the predictive performance of each index expressed as sensitivity, specificity, positive/negative predictive value, accuracy, positive/negative likelihood ratio, diagnostic odds ratio (DOR), $\mathrm{F}_{1}$ score, and Matthew's correlation coefficient (MCC).

We also performed a binary logistic regression analysis to derive variables independently associated with SBT failure from baseline clinical characteristics, comorbidities, and the candidate predictors computed from the ventilatory variables. Variables considered a priori clinically relevant, and variables with a $p$ value less than 0.2 in the bivariate analysis were included in the multivariable models (using forward selection). The model's goodness-of-fit was evaluated with the Hosmer \& Lemeshow test and Nagelkerke $\mathrm{R}^{2}$. We reported odds ratios (OR) with $95 \%$ confidence intervals $(95 \% \mathrm{CI})$.

Finally, Pearson's $r$ was used to correlate the index tests with SBT duration and $\mathrm{P}_{\mathrm{a}} \mathrm{CO}_{2}$ at the end of the weaning trial.

All tests were two-tailed; statistical significance was indicated by $p<0.05$. All analyses were performed using MedCalc statistical software version 19.2.5 (MedCalc Software Ltd, Ostend, Belgium). Further details on the statistical methods can be found in the supplementary information.

Ethics approval and consent to participate. The study was approved by the local institutional review board for human studies (Ethics Committee of the State Chamber of Physicians of Baden-Wuerttemberg, Germany, file number F-2018-116) and performed following the Declaration of Helsinki. Written informed consent was obtained from all patients or a legal representative.

Consent for publication. Not applicable.

\section{Results}

One-hundred and thirty-nine consecutive patients were screened between March 2019 and August 2020, of whom 130 (93.5\%) were included in the study. Nine patients were excluded from the analyses; three had a diagnosis of neuromuscular disease, four died before weaning completion, and two declined to participate.

Clinical characteristics differed between patients with successful and failed SBT regarding smoking history, presence of chronic obstructive pulmonary disease (COPD) and malignancy, and acute exacerbation of COPD as the primary reason for intubation with mechanical ventilation (Table 1).

Failure of the SBT occurred in 30 patients (23.1\%) (Supplementary Table S2), with significant differences observed in ventilatory variables and MP indices (Table 2).

Patients' baseline clinical characteristics and outcomes were equally distributed after each randomization for cross-validation (Supplementary Table S1). Supplementary Table S3 shows the mean threshold values derived from the training sets that best predicted SBT failure.

Absolute MP showed poor diagnostic accuracy (MCC 0.26; AUROC 0.68, 95\%CI [0.59-0.75], $p=0.002$ ), but there was a notable increase in discriminatory performance when MP was normalized to surrogates of lung volume (LTC dyn -MP: DOR 10.0, MCC 0.37, AUROC 0.76 [95\%CI 0.68-0.83], $p<0.001$; PBW-MP: DOR 5.3, MCC 0.29, AUROC 0.71 [0.62-0.79], $p<0.001$ ), further improved by correction for corresponding mechanical ventilation $\mathrm{P}_{\mathrm{a}} \mathrm{CO}_{2}$ (Power index $\mathrm{rs}$ : DOR 8.6, MCC 0.42, AUROC 0.81 [0.73-0.87], $p<0.001$ ) (Tables 3, 4, Fig. 2). Most of these indices predominantly showed a higher negative predictive value regarding failure of the SBT.

In the binary logistic regression analysis, the Power index (OR 1.48 per $1000 \mathrm{~cm} \mathrm{H}_{2} \mathrm{O}^{2} / \mathrm{min}, 95 \% \mathrm{CI}$ [1.24-1.76], $p<0.001)$ was independently associated with failure of the SBT in univariable, and its components $\mathrm{LTC}_{\mathrm{dyn}}-\mathrm{MP}\left(1.25\right.$ per $\left.1000 \mathrm{cmH}_{2} \mathrm{O}^{2} / \mathrm{min},[1.06-1.46], p<0.001\right)$ and mechanical ventilation $\mathrm{P}_{\mathrm{a}} \mathrm{CO}_{2}(1.17$ [1.06-1.28], $p<0.001)$ in the multivariable model $\left(\mathrm{R}^{2} 0.293\right)$ (Table 5, Supplementary Table S4-S6).

Finally, the Power index ${ }^{2.0}$ significantly correlated with the duration of SBT $(r=-0.38[-0.52$ to -0.22 ; $p<0.001]$ ) (Supplementary Table S7) and with $\mathrm{P}_{\mathrm{a}} \mathrm{CO}_{2}$ at end of the weaning trial $(r=0.57[0.43-0.67] ; p<0.001)$ (Supplementary Fig. S3). 


\begin{tabular}{|c|c|c|c|c|}
\hline Clinical characteristics & $\begin{array}{l}\text { All patients } \\
(n=130)\end{array}$ & $\begin{array}{l}\text { SBT success } \\
(\mathrm{n}=100)\end{array}$ & $\begin{array}{l}\text { SBT failure } \\
(\mathrm{n}=30)\end{array}$ & $p$ value $^{\mathrm{a}}$ \\
\hline Age (years) & $69(60-76)$ & $69(59-76)$ & $70(65-72)$ & $0.923^{\mathrm{c}}$ \\
\hline Gender (male) & $82(63.1)$ & $66(66.0)$ & $16(53.3)$ & $0.209^{\mathrm{d}}$ \\
\hline Body mass index $\left(\mathrm{kg} / \mathrm{m}^{2}\right)$ & $26.1(23.0-31.1)$ & $25.5(22.9-30.9)$ & $28.3(24.2-33.3)$ & $0.085^{c}$ \\
\hline Obesity $\left(\mathrm{BMI} \geq 30 \mathrm{~kg} / \mathrm{m}^{2}\right)$ & $40(30.8)$ & $28(28.0)$ & $12(40.0)$ & $0.213^{\mathrm{d}}$ \\
\hline Smoking history & $48(36.9)$ & $29(29.0)$ & $19(63.3)$ & $<0.001^{\mathrm{d}}$ \\
\hline APACHE-II (points) & $16(12-19)$ & $16(12-19)$ & $16(12-20)$ & $0.772^{\mathrm{b}}$ \\
\hline Albumin (g/dL) & $2.1(1.8-2.5)$ & $2.1(1.8-2.5)$ & $2.1(1.9-2.6)$ & $0.269^{c}$ \\
\hline Ventilator days on admission & $25(16-34)$ & $25(16-33)$ & $27(16-38)$ & $0.333^{\mathrm{c}}$ \\
\hline Intubation to tracheostomy (days) & $12(7-18)$ & $12(7-18)$ & $10(6-16)$ & $0.445^{c}$ \\
\hline ECLA & $14(10.8)$ & $13(13.0)$ & $1(3.3)$ & $0.136^{\mathrm{d}}$ \\
\hline \multicolumn{5}{|l|}{ Reason for mechanical ventilation } \\
\hline Pneumonia & $51(39.2)$ & $37(37.0)$ & $14(46.7)$ & $0.343^{\mathrm{d}}$ \\
\hline Surgery & $32(24.6)$ & $28(28.0)$ & $4(13.3)$ & $0.103^{\mathrm{d}}$ \\
\hline Cardiopulmonary resuscitation & $10(7.7)$ & $7(7.0)$ & $3(10.0)$ & $0.590^{\mathrm{d}}$ \\
\hline Acute exacerbation of COPD & $10(7.7)$ & $4(4.0)$ & $6(20.0)$ & $0.004^{d}$ \\
\hline Acute heart failure & $6(4.6)$ & $5(5.0)$ & $1(3.3)$ & $1.000^{\mathrm{e}}$ \\
\hline Sepsis (including septic shock) & $7(5.4)$ & $6(6.0)$ & $1(3.3)$ & $1.000^{\mathrm{e}}$ \\
\hline Other & $17(13.1)$ & $13(13.0)$ & $4(13.3)$ & $0.962^{\mathrm{d}}$ \\
\hline \multicolumn{5}{|l|}{ Comorbidities } \\
\hline Charlson comorbidity index (points) & $5(4-7)$ & $5(4-7)$ & $6(4-7)$ & $0.507^{\mathrm{c}}$ \\
\hline Renal insufficiency & $46(35.4)$ & $36(36.0)$ & $10(33.3)$ & $0.789^{\mathrm{d}}$ \\
\hline Hemodialysis & $24(18.5)$ & $19(19.0)$ & $5(16.7)$ & $0.678^{\mathrm{d}}$ \\
\hline Diabetes mellitus & $35(26.9)$ & $25(25.0)$ & $10(33.3)$ & $0.369^{\mathrm{d}}$ \\
\hline Coronary artery disease & $33(25.4)$ & $25(25.0)$ & $8(26.7)$ & $0.854^{\mathrm{d}}$ \\
\hline COPD & $30(23.1)$ & $16(16.0)$ & $14(46.7)$ & $<0.001^{\text {d }}$ \\
\hline Chronic heart failure & $17(13.1)$ & $15(15.0)$ & $2(6.7)$ & $0.234^{\mathrm{d}}$ \\
\hline Malignancy & $10(7.7)$ & $5(5.0)$ & $5(16.7)$ & $0.036^{d}$ \\
\hline Hepatopathy & $7(5.4)$ & $6(6.0)$ & $1(3.3)$ & $1.000^{\mathrm{e}}$ \\
\hline Interstitial lung disease & $8(6.2)$ & $7(7.0)$ & $1(3.3)$ & $0.681^{\mathrm{e}}$ \\
\hline
\end{tabular}

Table 1. Clinical characteristics on admission to the weaning center-comparison of patients with successful and unsuccessful first SBT. Continuous variables are presented as median (- interquartile range [IQR]; categorical variables are presented as number (\%). BMI, body mass index; APACHE-II, Acute Physiology and Chronic Health Evaluation II score; ECLA, extracorporeal lung assistance (during acute respiratory failure); COPD, chronic obstructive pulmonary disease. ${ }^{a} p$ value for differences between patients with successful and unsuccessful SBT. ${ }^{b}$ Student's $t$-test. ${ }^{\mathrm{c}}$ Mann-Whitney $U$-test. ${ }^{\mathrm{d}}$ Chi-squared test. ${ }^{\mathrm{e}}$ Fisher's exact test. Significant values are in Bold.

\section{Discussion}

The present analysis aimed to investigate the discriminatory performance of the mechanical power in predicting the outcome of a short weaning trial in prolonged ventilated patients. In summary, absolute MP performed poorly, with improvement by correction for surrogates of lung volume (e. g. LTC $_{\text {dyn }}$, so-called specific MP, consistent with stress intensity) and mechanical ventilation $\mathrm{P}_{\mathrm{a}} \mathrm{CO}_{2}$, the former allowing comparability between differences in gender and body height, the latter simulating isocapnic conditions by accounting for individual respiratory rate and ventilator pressure settings. Moreover, in the multivariable logistic regression analysis, these indices were independently associated with failure of the SBT.

This approach of combining mechanical ventilation stress intensity with a marker of alveolar ventilation is a simplified method for estimating associated mechanical power (stress intensity times LTC $_{\text {dyn }}$ ) required to defend alveolar ventilation and has recently been shown to correlate well with patients' ability to wean from prolonged ventilation successfully ${ }^{13}$. For instance, the difference between actual $\mathrm{LTC}_{\mathrm{dyn}}-\mathrm{MP}$ and Power index approximates the required change in ventilator stress intensity output (determined by the inspiratory pressures, PEEP, and respiratory rate) necessary to reach the target $\mathrm{P}_{\mathrm{a}} \mathrm{CO}_{2}$, which was arbitrary set at $45 \mathrm{mmHg}$ in the present study. As a prerequisite for this, we aimed at near totally unloading the respiratory pump by applying the same (high) inspiratory pressure during both controlled and assisted ventilation (in A/C mode), thereby minimizing patients' respiratory muscle activity between SBT. In other words, even if patients' respiratory muscles were relaxed, the applied backup respiratory rate and inspiratory pressure (both of which determine minute ventilation) were sufficient to defend alveolar ventilation. Nevertheless, our technique is a trade-off between the accuracy of MP measurement and its clinical applicability. This approach to weaning is contrary to the current practice of liberation attempts from short-term mechanical ventilation, which is a gradual reduction in ventilator support as 


\begin{tabular}{|c|c|c|c|c|}
\hline First SBT & $\begin{array}{l}\text { All patients } \\
(\mathrm{n}=130)\end{array}$ & $\begin{array}{l}\text { SBT success } \\
(\mathrm{n}=100)\end{array}$ & $\begin{array}{l}\text { SBT failure } \\
(\mathbf{n}=\mathbf{3 0})\end{array}$ & $p$ value $^{\mathrm{a}}$ \\
\hline Time from admission to first SBT (days) & $1(0-3)$ & $1(0-3)$ & $1(1-3)$ & $0.638^{\mathrm{b}}$ \\
\hline Hemoglobin on first SBT (g/dL) & $8.3( \pm 1.2)$ & $8.3( \pm 1.2)$ & $8.1( \pm 1.3)$ & $0.266^{\mathrm{c}}$ \\
\hline \multicolumn{5}{|l|}{ Ventilatory variables \& MP indices } \\
\hline $\mathrm{F}_{\mathrm{i}} \mathrm{O}_{2}$ & $0.27( \pm 0.05)$ & $0.27( \pm 0.05)$ & $0.28( \pm 0.04)$ & $0.156^{\mathrm{c}}$ \\
\hline Tidal volume $(\mathrm{mL})$ & $547( \pm 86)$ & $547( \pm 83)$ & $547( \pm 97)$ & $0.459^{\mathrm{c}}$ \\
\hline Minute ventilation (L/min) & $9.0( \pm 1.9)$ & $9.0( \pm 2.0)$ & $9.0( \pm 1.7)$ & $0.676^{\mathrm{c}}$ \\
\hline $\mathrm{P}_{\mathrm{a}} \mathrm{CO}_{2}$ on $\mathrm{MV}(\mathrm{mmHg})$ & $35.0( \pm 5.4)$ & $33.9( \pm 4.8)$ & $38.8( \pm 4.5)$ & $<0.001^{\mathrm{b}}$ \\
\hline Ventilatory ratio & $1.19( \pm 0.34)$ & $1.14( \pm 0.34)$ & $1.38( \pm 0.32)$ & $<0.001^{\mathrm{c}}$ \\
\hline PEEP $\left(\mathrm{cmH}_{2} \mathrm{O}\right)$ & $6.0( \pm 0.9)$ & $6.0( \pm 0.9)$ & $6.0( \pm 0.8)$ & $0.717^{\mathrm{c}}$ \\
\hline $\mathrm{P}_{\max }\left(\mathrm{cmH}_{2} \mathrm{O}\right)$ & $23.6( \pm 4.2)$ & $22.8( \pm 4.1)$ & $26.3( \pm 3.5)$ & $<0.001^{\mathrm{b}}$ \\
\hline$\Delta \mathrm{P}_{\mathrm{aw}}\left(\mathrm{cmH}_{2} \mathrm{O}\right)$ & $17.6( \pm 4.0)$ & $16.8( \pm 3.9)$ & $20.3( \pm 3.4)$ & $<0.001^{\mathrm{c}}$ \\
\hline $\mathrm{LTC}_{\mathrm{dyn}}\left(\mathrm{mL} / \mathrm{cmH}_{2} \mathrm{O}\right)$ & $32.8( \pm 9.6)$ & $34.3( \pm 9.6)$ & $27.9( \pm 7.9)$ & $<0.001^{\mathrm{c}}$ \\
\hline Mechanical power $(\mathrm{J} / \mathrm{min})$ & $21.0( \pm 5.9)$ & $20.2( \pm 5.7)$ & $23.6( \pm 5.8)$ & $0.004^{c}$ \\
\hline PBW-MP (J/min/kg) & $0.3295( \pm 0.1106)$ & $0.3111( \pm 0.1033)$ & $0.3907( \pm 0.1137)$ & $<0.001^{\mathrm{c}}$ \\
\hline $\mathrm{LTC}_{\mathrm{dyn}}-\mathrm{MP}\left(\mathrm{cmH}_{2} \mathrm{O}^{2} / \mathrm{min}\right)$ & $7167( \pm 3063)$ & $6569( \pm 2885)$ & $9159( \pm 2824)$ & $<0.001^{\mathrm{c}}$ \\
\hline Power index ${ }_{\mathrm{rs}}^{1.0}\left(\mathrm{cmH}_{2} \mathrm{O}^{2} / \mathrm{min}\right)$ & $5696( \pm 2884)$ & $5012( \pm 2490)$ & $7978( \pm 2971)$ & $<0.001^{\mathrm{c}}$ \\
\hline Power index ${ }_{\mathrm{rs}}^{2.0}\left(\mathrm{cmH}_{2} \mathrm{O}^{2} / \mathrm{min}\right)$ & $4636( \pm 2896)$ & $3902( \pm 2323)$ & $7082( \pm 3292)$ & $<0.001^{\mathrm{c}}$ \\
\hline \multicolumn{5}{|l|}{$\mathrm{P}_{\mathrm{a}} \mathrm{CO}_{2}$ on $\mathrm{ABG}$} \\
\hline $\mathrm{P}_{\mathrm{a}} \mathrm{CO}_{2}$ on $\mathrm{MV}$ pre-SBT $(\mathrm{mmHg})$ & $35.0( \pm 5.4)$ & $33.9( \pm 4.8)$ & $38.8( \pm 5.5)$ & $0.001^{\mathrm{b}}$ \\
\hline $\mathrm{P}_{\mathrm{a}} \mathrm{CO}_{2}$ on $\mathrm{SB}$ post-SBT $(\mathrm{mmHg})^{*}$ & $38.7( \pm 7.0)$ & $36.3( \pm 4.6)$ & $48.9( \pm 6.5)$ & $<0.001^{\mathrm{b}}$ \\
\hline$\Delta \mathrm{P}_{\mathrm{a}} \mathrm{CO}_{2}$ (post-/pre-SBT) & $3.9( \pm 5.8)$ & $2.4( \pm 4.4)$ & $10.2( \pm 7.2)$ & $<0.001^{\mathrm{c}}$ \\
\hline \multicolumn{5}{|l|}{$\mathrm{pH}$ on $\mathrm{ABG}$} \\
\hline $\mathrm{pH}$ on $\mathrm{MV}$ pre-SBT & $7.50( \pm 0.05)$ & $7.50( \pm 0.05)$ & $7.48( \pm 0.05)$ & $0.192^{\mathrm{c}}$ \\
\hline $\mathrm{pH}$ on $\mathrm{SB}$ post-SBT* & $7.46( \pm 0.06)$ & $7.48( \pm 0.04)$ & $7.40( \pm 0.07)$ & $<0.001^{\mathrm{c}}$ \\
\hline$\Delta \mathrm{pH}$ (post-/pre-SBT) & $-0.03( \pm 0.06)$ & $-0.02( \pm 0.05)$ & $-0.09( \pm 0.07)$ & $<0.001^{\mathrm{c}}$ \\
\hline \multicolumn{5}{|l|}{$\mathrm{P}_{\mathrm{a}} \mathrm{O}_{2}$ on $\mathrm{ABG}$} \\
\hline $\mathrm{P}_{\mathrm{a}} \mathrm{O}_{2}$ on $\mathrm{MV}$ pre-SBT $(\mathrm{mmHg})$ & $83.7( \pm 15.9)$ & $85.0( \pm 15.9)$ & $79.4( \pm 15.5)$ & $0.069^{c}$ \\
\hline $\mathrm{P}_{\mathrm{a}} \mathrm{O}_{2}$ on $\mathrm{SB}$ post-SBT $(\mathrm{mmHg})^{*}$ & $76.9( \pm 18.1)$ & $77.4( \pm 19.0)$ & $74.7( \pm 13.8)$ & $0.790^{\mathrm{c}}$ \\
\hline$\Delta \mathrm{P}_{\mathrm{a}} \mathrm{O}_{2}$ (post-/pre-SBT) & $-7.4( \pm 21.0)$ & $-7.6( \pm 22.2)$ & $-6.4( \pm 15.0)$ & $0.506^{\mathrm{c}}$ \\
\hline Duration of first SBT (min) & $30(30-30)$ & $30(30-30)$ & $30(11-30)$ & $<0.001^{\mathrm{c}}$ \\
\hline
\end{tabular}

Table 2. Results of first SBT_comparison of patients with successful and unsuccessful SBT. Continuous variables are presented as arithmetic means values ( \pm standard deviation) or median (- interquartile range [IQR]); categorical variables are presented as numbers (\%). SBT, spontaneous breathing trial; MP, mechanical power; $\mathrm{F}_{\mathrm{i}} \mathrm{O}_{2}$, the fraction of inspired oxygen; $\mathrm{MV}$, mechanical ventilation; PEEP, positive end-expiratory pressure; $\mathrm{P}_{\max }$, the pre-set inspiratory positive airway pressure; $\Delta \mathrm{P}_{\mathrm{aw}}$, dynamic driving pressure (defined as $\mathrm{P}_{\max }$-PEEP in the pressure-controlled ventilation mode); $\mathrm{LTC}_{\mathrm{dyn}}$, dynamic lung-thorax compliance; PBW-MP, mechanical power normalized to predicted body weight; $\mathrm{LTC}_{\mathrm{dyn}}-\mathrm{MP}$, mechanical power normalized to dynamic lung-thorax compliance, ABG, arterial blood gas analysis. *ABG at the end of the SBT was missing in seven patients due to severe respiratory distress, requiring immediate resumption of mechanical ventilation. ${ }^{\mathrm{a}} p$ value for differences between patients with successful and unsuccessful SBT. ${ }^{\mathrm{b}}$ Student's $t$-test. ${ }^{\mathrm{c}}$ Mann-Whitney $U$-test. ${ }^{\mathrm{d}}$ Chi-squared test. Significant values are in Bold.

soon as weaning readiness is suspected from clinical screening criteria (markers of respiratory system recovery), with an optional assessment of weaning predictors during a short period of spontaneous breathing, followed by a weaning trial ${ }^{14}$. Even so, our approach has been shown to speed up weaning in prolonged ventilated, tracheotomized patients compared to a gradual reduction of pressure support ${ }^{21}$.

Because no single parameter provides a perfect prediction for readiness testing, the combination of variables that indicate when to undertake or refrain from making the first weaning attempt is appealing ${ }^{4,7,22,23}$. Yet, most of the described integrative weaning indices (e.g., CROP, Integrative Weaning Index, or CORE) showed only moderate accuracy in predicting the outcome of a short weaning trial, and none of them were validated in prolonged ventilated patients. Moreover, since most of the included variables were measured during spontaneous breathing, assessment of these indices is more complex, reducing their applicability in daily routine. In contrast, $\mathrm{PI}_{\mathrm{rs}}$ can be assessed at the bedside without disconnecting the patient from the ventilator.

Weaning predictors are used as a decision point to determine whether a patient may advance to a spontaneous breathing trial. This approach has two main goals. First, it may help identify patients who are not yet ready for a weaning trial, assuming a failed SBT results in worse outcomes. However, in contrast to extubation failure, which is associated with increased mortality ${ }^{24}$, there is so far no evidence that a failed weaning trial could be harmful, provided ventilation is resumed immediately in the event of respiratory distress. In a physiologic study, Laghi 


\begin{tabular}{|c|c|c|c|c|c|c|c|c|c|c|}
\hline \multirow[b]{2}{*}{ Variables } & \multicolumn{10}{|c|}{ Failure of spontaneous breathing trial } \\
\hline & Sens & Spec & PPV & NPV & Accuracy & PLR & NLR & DOR & $F_{1}$ & MCC \\
\hline $\mathrm{P}_{\mathrm{a}} \mathrm{CO}_{2}$ on $\mathrm{MV}$ & $68(40-89)$ & $69(54-81)$ & $41(28-54)$ & $88(77-94)$ & $68(56-79)$ & $2.4(1.3-4.2)$ & $0.5(1.0-0.2)$ & 5.6 & 0.51 & 0.32 \\
\hline Ventilatory ratio & $68(41-88)$ & $70(55-83)$ & $40(28-54)$ & $88(78-94)$ & $69(62-80)$ & $2.3(1.3-4.0)$ & $0.4(1.0-0.2)$ & 6.9 & 0.50 & 0.33 \\
\hline LTC $_{\text {dyn }}$ & $63(37-84)$ & $69(55-81)$ & $37(25-52)$ & $87(77-93)$ & $68(55-79)$ & $2.0(1.2-3.6)$ & $0.5(1.0-0.3)$ & 5.1 & 0.46 & 0.28 \\
\hline Mechanical power & $70(44-88)$ & $61(46-74)$ & $34(24-46)$ & $88(76-94)$ & $63(50-74)$ & $1.7(1.1-2.8)$ & $0.5(1.1-0.2)$ & 6.7 & 0.45 & 0.26 \\
\hline PBW-MP & $65(39-85)$ & $67(53-79)$ & $39(26-53)$ & $87(76-93)$ & 67 (54-78) & $2.3(1.2-4.6)$ & $0.5(1.0-0.3)$ & 5.3 & 0.47 & 0.29 \\
\hline $\mathrm{LTC}_{\mathrm{dyn}}-\mathrm{MP}$ & $72(45-89)$ & $71(57-83)$ & $43(30-56)$ & 90 (79-95) & $71(59-82)$ & $2.5(1.2-4.4)$ & $0.4(0.9-0.2)$ & 10.0 & 0.53 & 0.37 \\
\hline Power index ${ }_{\mathrm{rs}}^{1.0}$ & 78 (47-93) & $72(58-84)$ & $45(32-58)$ & $91(80-96)$ & $72(60-83)$ & $2.7(1.6-4.8)$ & $0.3(0.8-0.1)$ & 8.6 & 0.57 & 0.42 \\
\hline Power index ${ }_{\mathrm{rs}}^{2.0}$ & 70 (42-90) & $75(60-86)$ & $46(32-60)$ & 89 (79-95) & $73(61-84)$ & $2.9(1.6-5.3)$ & $0.4(0.9-0.2)$ & 7.1 & 0.55 & 0.39 \\
\hline
\end{tabular}

Table 3. Cross-validated performance of variables analyzed to predict the outcome of SBT-mean values derived from the test sets. Assessment of mean sensitivity and specificity, positive and negative predictive value, positive and negative likelihood ratio, diagnostic odds ratio, $\mathrm{F}_{1}$ score, and Matthews correlation coefficient (with 95\% confidenceintervals). SBT, spontaneous breathing trial; Sens, sensitivity; Spec, specificity; PPV, positive predictive value; NPV, negative predictive value; PLR, positive likelihood ratio; NLR, negative likelihood ratio; DOR, diagnostic odds ratio; $\mathrm{F}_{1}, \mathrm{~F}_{1}$ score; $\mathrm{MCC}$, Matthews correlation coefficient; $\mathrm{MV}$, mechanical ventilation; $\mathrm{LTC}_{\mathrm{dyn}}$, dynamic lung-thorax compliance; PBW-MP, mechanical power normalized to predicted body weight; $\mathrm{LTC}_{\mathrm{dyn}}$-MP, mechanical power normalized to dynamic lung-thorax compliance.

\begin{tabular}{|l|l|}
\hline Variables & AUROC \\
\hline $\mathrm{P}_{\mathrm{a}} \mathrm{CO}_{2}$ on MV & $0.74(0.66-0.81)$ \\
\hline Ventilatory ratio & $0.74(0.65-0.81)$ \\
\hline $\mathrm{LTC}_{\mathrm{dyn}}$ & $0.71(0.62-0.78)$ \\
\hline Mechanical power & $0.68(0.59-0.75)$ \\
\hline PBW-MP & $0.71(0.62-0.79)$ \\
\hline LTC $_{\mathrm{dyn}}-\mathrm{MP}$ & $0.76(0.68-0.83)$ \\
\hline Power index $_{\mathrm{rs}}^{1.0}$ & $0.81(0.73-0.87)$ \\
\hline Power index $_{\mathrm{rs}}^{2.0}$ & $0.81(0.73-0.87)$ \\
\hline
\end{tabular}

Table 4. The area under the ROC curve for each variable analyzed to predict the outcome of first SBT-all patients. The accuracy of each variable in the whole study population presented as the area under the ROC curve with 95\% confidence intervals. SBT, spontaneous breathing trial; MV, mechanical ventilation; $\mathrm{LTC}_{\mathrm{dyn}}$, dynamic lung-thorax compliance; PBW-MP, mechanical power normalized to predicted body weight; $\mathrm{LTC}_{\mathrm{dyn}}-\mathrm{MP}$, mechanical power normalized to dynamic lung-thorax compliance.

and co-workers demonstrated that patients who failed a weaning trial did not develop contractile fatigue of the diaphragm (consistent with muscle injury), potentially leading to subsequent unsuccessful weaning attempts or unsuccessful liberation from the ventilator ${ }^{25}$. Moreover, these investigators were able to show that diaphragmatic recruitment at the end of a failed weaning trial, estimated by the electrical activity of the diaphragm, was half of the maximum, corresponding to reflex inhibition of the diaphragm with at the same time redistribution of motoneuron output to rib cage and accessory muscles, a potential mechanism for the prevention of diaphragmatic contractile fatigue ${ }^{26}$. Accordingly, patients categorized as difficult to wean, failing up to three SBT before successful discontinuation of mechanical ventilation, showed no increase in ICU- or hospital mortality compared to patients successfully extubated on the first attempt ${ }^{27,28}$. The second and presumably more important goal of using weaning predictors is to avoid unnecessarily prolonged ventilation, which associates with significant morbidity and mortality ${ }^{1,2}$. In this context, given its higher negative predictive value, the Power index may be particularly useful in identifying patients who are likely to succeed in a trial of spontaneous breathing rather than indicating weaning failure. This finding is in line with most classic prediction variables, which display high sensitivity for weaning success (e. g., the rapid shallow breathing index or maximum inspiratory pressure), but usually lack sufficient specificity ${ }^{4}$. This phenomenon may, in part, be explained by the duration of weaning trials in different studies. Since the relationship between critical stress imposed on respiratory muscles and time to task failure in healthy subjects follows an inverse power function ${ }^{29}, 30 \mathrm{~min}$ of spontaneous breathing may be too short for some patients for the development of apparent respiratory distress or alveolar hypoventilation, heightened muscle effort is not sustained for a sufficient time ${ }^{25}$. Yet, these patients may experience weaning failure in a more extended trial $^{25}$. Accordingly, in the present study, the Power index was significantly correlated with the duration of SBT. Nevertheless, the selected threshold value finally determines LTC $_{\mathrm{dyn}}-\mathrm{MP}$ 's predictive weaning outcome ability, allowing further adjustments depending on the primary goal of using it as a weaning predictor (e.g., preferring a higher sensitivity or specificity for SBT failure).

In a recent observational study, patients determined to have premature or delayed weaning trials, compared to patients with an opportune SBT, showed worse clinical outcomes ${ }^{30}$. However, so far, controlled studies have 


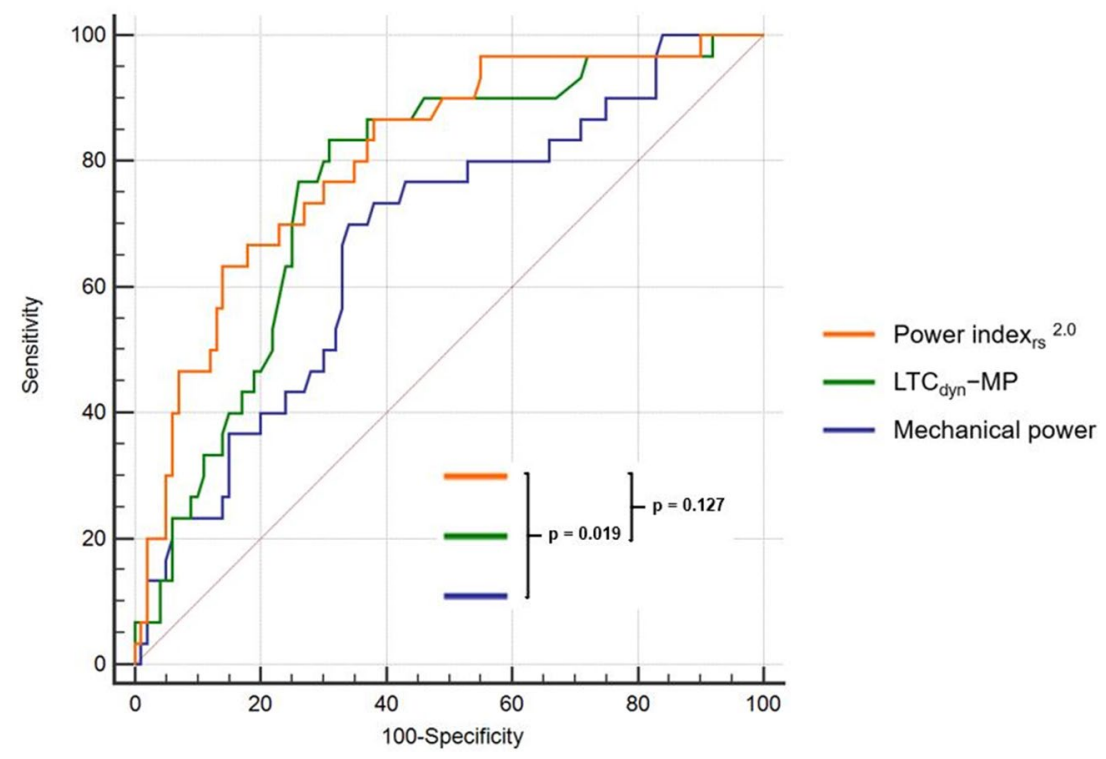

Figure 2. Comparison of ROC curves for mechanical power, $\mathrm{LTC}_{\mathrm{dyn}}-\mathrm{MP}$, and the power index ${ }_{\mathrm{rs}}^{2.0}$ predicting the outcome of SBT - all patients. Abbreviations: ROC, receiver operating characteristic curve; $\mathrm{LTC}_{\mathrm{dyn}}-\mathrm{MP}$, mechanical power normalized to dynamic lung-thorax compliance.

\begin{tabular}{|c|c|c|c|c|c|c|c|c|}
\hline \multirow[b]{2}{*}{ Variables } & \multicolumn{2}{|c|}{ Univariable analysis } & \multicolumn{2}{|c|}{ Multivariable model 1} & \multicolumn{2}{|c|}{ Multivariable model 2} & \multicolumn{2}{|c|}{ Multivariable model 3} \\
\hline & \begin{tabular}{|l|} 
OR $(95 \% \mathrm{CI})$ \\
\end{tabular} & $P$ & OR $(95 \% \mathrm{CI})$ & $P$ & OR $(95 \% \mathrm{CI})$ & $P$ & OR $(95 \% \mathrm{CI})$ & $P$ \\
\hline Age & $1.00(0.97-1.03)$ & 0.978 & & & & & & \\
\hline Gender (male) & $0.59(0.26-1.35)$ & 0.210 & & & & & & \\
\hline BMI & $1.04(0.98-1.10)$ & 0.166 & & & & & & \\
\hline APACHE-II & $1.01(0.94-1.09)$ & 0.770 & & & & & & \\
\hline CCI & $1.07(0.89-1.28)$ & 0.472 & & & & & & \\
\hline COPD & $4.5(1.88-11.24)$ & $<0.01$ & & & & & & \\
\hline Obesity & $1.71(0.73-4.01)$ & 0.214 & & & & & & \\
\hline Immunosuppression & $0.51(0.14-1.85)$ & 0.304 & & & & & & \\
\hline Malignancy & $3.80(1.02-14.2)$ & 0.047 & & & & & & \\
\hline VD on admission & $1.01(0.99-1.03)$ & 0.174 & & & & & & \\
\hline ECLA & $0.23(0.03-1.84)$ & 0.166 & & & & & & \\
\hline $\mathrm{Hb}$ on SBT & $0.99(0.95-1.02)$ & 0.460 & & & & & & \\
\hline $\mathrm{P}_{\mathrm{a}} \mathrm{CO}_{2}$ on $\mathrm{MV}$ & $1.21(1.10-1.33)$ & $<0.01$ & $1.19(1.09-1.31)$ & $<0.01$ & $1.30(1.14-1.47)$ & $<0.01$ & $1.17(1.06-1.28)$ & $<0.01$ \\
\hline Ventilatory ratio & $7.47(1.88-29.6)$ & $<0.01$ & & & $0.08(0.01-0.92)$ & 0.043 & & \\
\hline LTC $_{d y n}$ & $0.91(0.86-0.97)$ & $<0.01$ & & & & & & \\
\hline Mechanical power & $1.10(1.03-1.19)$ & $<0.01$ & $1.09(1.00-1.18)$ & 0.043 & & & & \\
\hline $\begin{array}{l}\text { PBW-MP, per } 10^{-2} \mathrm{~J} / \\
\mathrm{min} / \mathrm{kg}\end{array}$ & $1.06(1.03-1.11)$ & $<0.01$ & & & $1.13(1.05-1.21)$ & $<0.01$ & & \\
\hline $\begin{array}{l}\text { LTC }_{\text {dyn }}-\mathrm{MP} \text {, per } 1000 \\
\mathrm{cmH}_{2} \mathrm{O}^{2} / \mathrm{min}\end{array}$ & $1.32(1.14-1.53)$ & $<0.01$ & & & & & $1.25(1.06-1.46)$ & $<0.01$ \\
\hline $\begin{array}{l}\text { Power index } \mathrm{rs}_{\mathrm{rs}}^{1.0} \text {, per } \\
1000 \mathrm{cmH}_{2} \mathrm{O}^{2} / \mathrm{min}\end{array}$ & $1.45(1.22-1.72)$ & $<0.01$ & & & & & & \\
\hline $\begin{array}{l}\text { Power index } \mathrm{rs}_{\mathrm{rs}}^{2.0} \text {, per } \\
1000 \mathrm{cmH}_{2} \mathrm{O}^{2} / \mathrm{min}\end{array}$ & $1.48(1.24-1.76)$ & $<0.01$ & & & & & & \\
\hline
\end{tabular}

Table 5. Variables associated with failure of SBT-Results of binary logistic regression analysis. Multivariable regression models included age, gender, BMI, COPD, malignancy, ventilator days on admission, ECLA, ventilatory ratio, and indices derived from calculated mechanical power. BMI, body mass index; APACHE-II, Acute Physiology and Chronic Health Evaluation II score; CCI, Charlson comorbidity index; COPD, chronic obstructive pulmonary disease; VD, ventilator days; ECLA, extracorporeal lung assistance; Hb, hemoglobin; SBT, spontaneous breathing trial; $\mathrm{MV}$, mechanical ventilation; $\mathrm{LTC}_{\mathrm{dyn}}$, dynamic lung-thorax compliance; PBW-MP, mechanical power normalized to predicted body weight; $\mathrm{LTC}_{\mathrm{dyn}}-\mathrm{MP}$, mechanical power normalized to dynamic lung-thorax compliance. Significant values are in Bold. 
failed to prove advantages of using weaning predictors (e. g., shortening the duration of mechanical ventilation) compared to decision making based on clinical screening criteria alone ${ }^{31}$. In two randomized trials, incorporating the rapid shallow breathing index (frequency-tidal volume ratio) in a weaning protocol, which was then used (intervention) or not used (control) for the decision to proceed on spontaneous breathing, this approach significantly prolonged weaning time, had no perceptible effect on mortality or extubation failure rates ${ }^{32}$, and thus may not be helpful in a weaning protocol ${ }^{33}$. Consequently, incorporating weaning predictors in the decision to proceed on a weaning trial generally has been questioned ${ }^{34}$, and routine use of such prediction variables is currently not recommended ${ }^{14,35}$.

Weaning and extubation outcomes in intubated patients are two independent processes, the inability to breathe without ventilator assistance due to an imbalance between load and capacity of respiratory muscles (referred to as non-airway failure or weaning failure) and the inability to maintain a patent airway (so-called airway failure $)^{36}$, both of which are associated with different risk factors ${ }^{37}$. This is a significant problem when assessing prediction variables in such patients, limiting the development of a single prediction model with high diagnostic accuracy. Tracheostomy protects the airway during both mechanical and self-ventilation, allowing the evaluation of spontaneous breathing in isolation. Hence, investigating such patients and comparing those who can or cannot defend alveolar ventilation may provide a deeper understanding of why weaning failure occurs.

Our study has several limitations. First, since it was a monocentric analysis, generalizability to other centers (external validity) is uncertain. Second, the first weaning attempt was made over $30 \mathrm{~min}$, which may have been too short, and a more extended trial may have disclosed more patients with weaning failure, affecting diagnostic accuracy of the prediction variables. Finally, since we evaluated only patients with tracheotomies, we cannot extrapolate our results to intubated patients.

\section{Conclusions}

Mechanical power normalized to lung-thorax compliance independently predicted the outcome of a short weaning trial and thus may serve for readiness testing before proceeding on spontaneous breathing in prolonged ventilated patients. Given its higher negative predictive value, this index may be particularly useful in identifying patients ready to attempt spontaneous breathing, thereby probably decreasing ventilation time.

\section{Data availability}

The datasets used and analyzed during the current study are available from the corresponding author on reasonable request.

Received: 13 August 2021; Accepted: 13 December 2021

Published online: 07 January 2022

\section{References}

1. Torres, A. et al. Re-intubation increases the risk of nosocomial pneumonia in patients needing mechanical ventilation. Am. J. Respir. Crit. Care Med. 152(1), 137-141 (1995).

2. Girou, E. et al. Association of non-invasive ventilation with nosocomial infections and survival in critically ill patients. JAMA 284(18), 2361-2367 (2000).

3. Ely, E. W. et al. Effect on the duration of mechanical ventilation of identifying patients capable of breathing spontaneously. N. Engl. J. Med. 335(25), 1864-1869 (1996).

4. Yang, K. L. \& Tobin, M. J. A prospective study of indexes predicting the outcome of trials of weaning from mechanical ventilation. N. Engl. J. Med. 324(21), 1445-1450 (1991).

5. Vallverdu, I. et al. Clinical characteristics, respiratory functional parameters, and outcome of a two-hour T-piece trial in patients weaning from mechanical ventilation. Am. J. Respir. Crit. Care Med. 158(6), 1855-1862 (1998).

6. Baptistella, A. F. et al. Predictive factors of weaning from mechanical ventilation and extubation outcome: A systematic review. J. Crit. Care. 48, 56-62 (2018).

7. Jubran, A., Grant, B. J. B., Laghi, F., Parthasarathy, S. \& Tobin, M. J. Weaning prediction: Esophageal pressure monitoring complements readiness testing. Am. J. Respir. Crit. Care. Med. 171(11), 1252-1259 (2005).

8. Vassilakopoulos, T., Zakynthinos, S. \& Roussos, C. The tension-time index and the frequency/tidal volume ratio are the major pathophysiologic determinants of weaning failure and success. Am. J. Respir. Crit. Care Med. 158(2), 378-385 (1998).

9. Purro, A. et al. Physiologic determinants of ventilator dependence in long-term mechanically ventilated patients. Am. J. Respir. Crit. Care Med. 161(4), 1115-1123 (2000).

10. Jubran, A. \& Tobin, M. J. Pathophysiologic basis of acute respiratory distress in patients who fail a trial of weaning from mechanical ventilation. Am. J. Respir. Crit. Care Med. 155(3), 906-915 (1997).

11. Gattinoni, L. et al. Ventilator-related causes of lung injury: the mechanical power. Intensive Care Med. 42(10), 1567-1575 (2016).

12. Ghiani, A. et al. Variables predicting weaning outcome in prolonged mechanically ventilated tracheotomized patients: A retrospective study. J. Intensive Care 8, 19 (2020).

13. Ghiani, A., Paderewska, J., Walcher, S. \& Neurohr, C. Mechanical power normalized to lung-thorax compliance predicts prolonged ventilation weaning failure: a prospective study. BMC Pulm. Med. 21, 202 (2021).

14. Boles, J. M. et al. Weaning from mechanical ventilation. Eur. Respir. J. 29(5), 1033-1056 (2007).

15. Okabe, Y. et al. Lung-thorax compliance measured during a spontaneous breathing trial is a good index of extubation failure in the surgical intensive care unit: a retrospective cohort study. J. Intensive Care 6, 44 (2018).

16. Becher, T., van der Staay, M., Schädler, D., Frerichs, I. \& Weiler, N. Calculation of mechanical power for pressure-controlled ventilation. Intensive Care Med. 45(9), 1321-1323 (2019).

17. Chiumello, D. et al. Bedside calculation of mechanical power during volume- and pressure-controlled mechanical ventilation. Crit. Care 24, 417 (2020).

18. Sinha, P., Fauvel, N. J., Singh, P. \& Soni, N. Analysis of ventilatory ratio as a novel method to monitor ventilatory adequacy at the bedside. Crit. Care 17, R34 (2013).

19. Brower, R. G. et al. Ventilation with lower tidal volumes as compared with traditional tidal volumes for acute lung injury and the acute respiratory distress syndrome. N. Engl. J. Med. 342(18), 1301-1308 (2000).

20. Arlot, S. \& Celisse, A. A survey of cross-validation procedures for model selection. Stat. Surv. 4, 40-79 (2010). 
21. Jubran, A. et al. Effect of pressure support vs unassisted breathing through a tracheostomy collar on weaning duration in patients requiring prolonged mechanical ventilation: A randomized trial. JAMA 309(7), 671-677 (2013).

22. Nemer, S. N. et al. A new integrative weaning index of discontinuation from mechanical ventilation. Crit. Care 13, R152 (2009).

23. Delisle, S. et al. Preliminary evaluation of a new index to predict the outcome of a spontaneous breathing trial. Respir. Care 56(10), 1500-1505 (2011).

24. Epstein, S. K., Ciubotaru, R. L. \& Wong, J. B. Effect of failed extubation on the outcome of mechanical ventilation. Chest 112(1), 186-192 (1997).

25. Laghi, F. et al. Is weaning failure caused by low-frequency fatigue of diaphragm?. Am. J. Respir. Crit. Care Med. 167(2), 120-127 (2003).

26. Laghi, F. et al. Inhibition of central activation of the diaphragm: A mechanism of weaning failure. J. Appl. Physiol. 129(2), 366-376 (2020).

27. Funk, G. C. et al. Incidence and outcome of weaning from mechanical ventilation according to new categories. Eur. Respir. J. 35(1), 88-94 (2010).

28. Penuelas, O. et al. Characteristics and outcomes of ventilated patients according to time to liberation from mechanical ventilation. Am. J. Respir. Crit. Care Med. 184(4), 430-437 (2011).

29. Bellemare, F. \& Grassino, A. Effect of pressure and timing of contraction on human diaphragm fatigue. J. Appl. Physiol. 53(5), $1190-1195$ (1982).

30. Diaz-Soto, M. et al. Premature, opportune, and delayed weaning in mechanically ventilated patients: A call for implementation of weaning protocols in low- and middle income coutries. Crit. Care Med. 48(5), 673-679 (2020).

31. Girard, T. D. et al. Efficacy and safety of paired sedation and ventilator weaning protocol for mechanically ventilated patients in intensive care (Awakening and Breathing Controlled trial): A randomized controlled trial. Lancet 371(9607), 126-134 (2008).

32. Tanios, M. A. et al. A randomized, controlled trial of the role of weaning predictors in clinical decision making. Crit. Care Med. 34(10), 2530-2535 (2006).

33. Figuerora-Casas, J. B. et al. Effect of using the rapid shallow breathing index as readiness criterion for spontaneous breathing trials in a weaning protocol. Am. J. Med. Sci. 359(2), 117-122 (2020).

34. Epstein, S. K. Routine use of weaning predictors: Not so fast. Crit. Care 13(5), 197 (2009).

35. Schmidt, G. A. et al. Official executive summary of an American Thoracic Society/American College of Chest Physicians clinical practice guideline: Liberation from mechanical ventilation in critically ill adults. Am. J. Respir. Crit. Care Med. 195(1), 115-119 (2017).

36. Epstein, S. K. \& Ciubotaru, R. L. Independent effects of etiology of failure and time to reintubation on outcome for patients failing extubation. Am. J. Respir. Crit. Care Med. 158(2), 489-493 (1998).

37. Jaber, S. et al. Risk factors and outcomes for airway failure versus non-airway failure int the intensive care unit: a multicenter observational study of 1514 extubation procedures. Crit. Care 22(1), 236 (2018).

\section{Author contributions}

Study concept and design: A.G. Acquisition of data: A.G., J.P., S.W. Analysis, and interpretation of data: A.G., J.P., S.W., K.T., C.N., N.K. First draft of the manuscript: A.G. Critical revision of the manuscript for important intellectual content: A.G., J.P., S.W., K.T., C.N., N.K. Statistical analysis: A.G. All authors have read and approved the final version of the manuscript.

\section{Competing interests}

The authors declare no competing interests.

\section{Additional information}

Supplementary Information The online version contains supplementary material available at https://doi.org/ 10.1038/s41598-021-03960-y.

Correspondence and requests for materials should be addressed to A.G.

Reprints and permissions information is available at www.nature.com/reprints.

Publisher's note Springer Nature remains neutral with regard to jurisdictional claims in published maps and institutional affiliations.

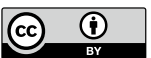

Open Access This article is licensed under a Creative Commons Attribution 4.0 International License, which permits use, sharing, adaptation, distribution and reproduction in any medium or format, as long as you give appropriate credit to the original author(s) and the source, provide a link to the Creative Commons licence, and indicate if changes were made. The images or other third party material in this article are included in the article's Creative Commons licence, unless indicated otherwise in a credit line to the material. If material is not included in the article's Creative Commons licence and your intended use is not permitted by statutory regulation or exceeds the permitted use, you will need to obtain permission directly from the copyright holder. To view a copy of this licence, visit http://creativecommons.org/licenses/by/4.0/.

(C) The Author(s) 2022 